\title{
O tema desastre na área da saúde: perfil da produção técnico-científica na base de dados especializada em desastres da Biblioteca Virtual em Saúde (BVS)
}

\author{
The theme of disaster in health care: profile of technical \\ and scientific production in the specialized database \\ on disasters of the Virtual Health Library - VHL
}

\begin{abstract}
Vania Rocha ${ }^{1}$
Elisa Francioli Ximenes ${ }^{1}$

Mauren Lopes de Carvalho ${ }^{1}$

Tais de Moura Ariza Alpino ${ }^{1}$

Carlos Machado de Freitas ${ }^{1}$
\end{abstract}

${ }^{1}$ Centro de Estudos e Pesquisas em Emergências e Desastres em Saúde, Fundação Oswaldo Cruz. R. Leopoldo Bulhões 1480 Manguinhos. 21.041-210 Rio de Janeiro RJ Brasil. vaniarocha@globomail.com
Abstract In the specialized database of the Virtual Health Library (VHL), the DISASTER database highlights the importance of the theme for the health sector. The scope of this article is to identify the profiles of technical and scientific publications in the specialized database. Based on systematic searches and the analysis of results it is possible to determine: the type of publication; the main topics addressed; the most common type of disasters mentioned in published materials, countries and regions as subjects, historic periods with the most publications and the current trend of publications. When examining the specialized data in detail, it soon becomes clear that the number of major topics is very high, making a specific search process in this database a challenging exercise. On the other hand, it is encouraging that the disaster topic is discussed and assessed in a broad and diversified manner, associated with different aspects of the natural and social sciences. The disaster issue requires the production of interdisciplinary knowledge development to reduce the impacts of disasters and for risk management. In this way, since the health sector is a interdisciplinary area, it can contribute to knowledge production.

Key words Disasters, Public health, Scientific production, VHL (Virtual Health Library)
Resumo Dentre as bases especializadas da Biblioteca Virtual em Saúde (BVS), a sobre "Desastres" indica a importância do tema para a área da saúde. O objetivo deste artigo foi identificar o perfil das publicações técnico-científicas desta base especializada. A partir de buscas sistemáticas e da análise dos resultados foi possível determinar: $o$ tipo de publicação, os principais assuntos abordados, os tipos de desastres mais frequentes nas publicações, os países e as regiões como assunto, período histórico com mais publicações e a tendência atual das publicações. Ao explorar detalhadamente a base especializada, percebe-se que o número de assuntos principais é elevado, característica que dificulta buscas específicas dentro dela. Por outro lado, é positivo o fato de o tema desastre ser tratado de forma ampla e diversificada, associado a diferentes assuntos das ciências naturais e das ciências sociais. O tema desastre exige produção de conhecimento interdisciplinar para reduzir os impactos dos desastres e para gestão dos riscos. Deste modo, a saúde, por se tratar de uma área interdisciplinar, pode contribuir para tal produção.

Palavras-chave Desastres, Saúde pública, Produção científica, BVS (Biblioteca Virtual em Saúde) 


\section{Introdução}

Na década de 90, com a popularização da internet abriu-se a possibilidade de acesso direto dos usuários à grande quantidade de fontes de dados e informações, surgindo a partir daí as bibliotecas virtuais como a Biblioteca Virtual em Saúde (BVS). É nesta mesma década de 90 que a temática ambiental ganhou proeminência, com a Rio 92, bem como a temática dos desastres com a Década Internacional de Redução de Desastres, tendo sido criado no final da mesma o Escritório Regional das Américas para a Redução de Desastres. Este foi um contexto favorável para que a BVS criasse algumas bases de dados especializadas, estando dentre elas a base "Desastres", agregando, compartilhando e facilitando o acesso ao conhecimento e informações, de modo a fortalecer as capacidades regionais de redução de desastres e seus impactos sobre a saúde no continente americano, o segundo mais afetado em número de desastres e fatalidades e o primeiro em impacto, contribuindo assim para o enfrentamento dos desafios relacionados ao tema.

O objetivo deste artigo é analisar os potenciais e limites da "Desastres", um importante bem público que disponibiliza diferentes tipos de informações sobre desastres, com foco na América Latina e Caribe (AL\&C). Procura-se atingir este objetivo através da identificação do perfil das publicações técnico-científicas, para determinar o tipo de literatura publicada, os principais assuntos, tipos de desastres abordados com maior frequência, países e regiões com maior número de investigações, período histórico com mais publicações e a tendência atual das publicações. Além disso, pretende-se situar neste contexto a literatura publicada no idioma português, verificando a sua participação na construção do conhecimento técnico-científico em desastres.

\section{Breve histórico da BVS Desastres}

Tão importante quanto produzir conhecimento em saúde é reunir e disponibilizar tais informações de forma eficiente e democrática. Em 1967, ainda no contexto em que não havia a ferramenta da internet possibilitando a operação direta das fontes de informação online pelos usuários, a Biblioteca Regional de Medicina (Bireme) foi estabelecida com o objetivo de estimular, fortalecer e ampliar o fluxo de informações científicas e técnicas no campo da medicina para os países da América Latina e Caribe (AL\&C) ${ }^{1}$.
A criação da Bireme foi fruto de um convênio entre a Organização Pan-americana de Saúde (OPAS), outras instituições de saúde e educação latino-americanas e a biblioteca nacional de medicina norte-americana, sendo a Universidade Federal de São Paulo, no Brasil, sede da infraestrutura física da instituição ${ }^{1}$. Logo tornou-se um ponto de contato entre a produção norte-americana e europeia em saúde e os profissionais de saúde e cientistas latino-americanos ${ }^{2}$.

Inicialmente, o conteúdo da Bireme consistia basicamente de artigos científicos da literatura biomédica, com tendência à superespecialização. No final dos anos 70 ocorre uma mudança na direção, que passa a adotar uma perspectiva mais abrangente. São introduzidos novos conteúdos (ciências sociais, economia, administração, planejamento em saúde), bem como novos estoques de informação (documentos, relatórios técnicos, pareceres e programas) ${ }^{3}$.

Em 1982, a Bireme passou a chamar-se Centro Latino-Americano e do Caribe de Informação em Ciências da Saúde, expressando melhor a sua missão: reunir e facilitar a disponibilização de informações em ciências da saúde através de um trabalho em rede, descentralizando o conhecimento em saúde, fortalecendo as capacidades locais e compartilhando recursos de informação entre os países da AL\&C. Sua sigla, porém, permaneceu a mesma ${ }^{1}$.

Com a popularização da internet, possibilitando o acesso direto do usuário às fontes de informação, em 1998, surge a BVS, o componente virtual da Bireme, com o objetivo de estimular o processo de expansão da informação como base da atividade científica e educacional em saúde ${ }^{1,2}$.

Atualmente, a BVS possui sete bases de dados especializadas, dentre elas a base "Desastres", a qual faz parte da coleção de bases de dados desde o início da mesma. O Centro Regional de Información sobre Desastres para América Latina y el Caribe - CRID é a instituição da Rede BVS responsável pela base "Desastres" e define os critérios de inclusão dos documentos na mesma ${ }^{4}$.

A existência da base "Desastres" indica a importância do tema, especialmente na década de 1990, considerada a década de redução de risco de desastres no contexto do aumento da frequência e intensidade destes eventos no mundo, segundo o Center for Research on the Epidemiology of Disasters (CRED) ${ }^{5}$. A importância da "Desastres” encontra-se em promover um bem público (o acesso ao conhecimento) regional, combinado com a necessidade de atingir diferentes públicos (especialistas e não especialistas) através da di- 
versidade de tipos de documentos (artigos, monografias, materiais de congressos e conferências, documentos de projeto, literatura "cinzenta", entre outros), valorizando a produção e idiomas dos países da AL\&C em um tema ainda pouco estudado, particularmente no âmbito da saúde.

No ano de 2014, a "Desastres" passou a integrar o portal "BVS Preparação e resposta aos Desastres", junto com outras bases, como Lilacs, Medline, Paho, Wholis e Biblioteca Cochrane, além de incluir recursos de internet, mantendo sua importância como bem público dentro do mesmo.

\section{Metodologia}

Para a análise da produção técnico-científica sobre desastres foi utilizada a base especializada "Desastres", que contém o universo de registros da produção internacional, regional e nacional, reunindo, principalmente, a produção da $\mathrm{AL} \& \mathrm{C}$ sobre o tema.

O portal de pesquisa da BVS (http://bvsalud. org/) permite a utilização de filtros para a identificação de documentos de interesse, utilizados conforme critérios escolhidos por quem faz a busca: Texto completo, Coleções, Base de dados, Assunto principal, Tipo de estudo, Aspecto clínico, Limite, País/Região como assunto, Idioma, Ano de publicação e Tipo de documento.

No dia 24 de setembro de 2013 havia 19.170 publicações dentro da base "Desastres". Foram escolhidos os cinco filtros que em conjunto poderiam estabelecer o perfil das publicações sobre desastres na BVS: Assunto principal, País/Região como assunto, Idioma, Ano de publicação e Tipo de documento. O filtro Assunto principal foi utilizado como chave de busca, enquanto os outros filtros auxiliaram na compreensão do perfil das publicações sobre desastres na BVS (Figura 1).

Ao utilizar o filtro Assunto principal, foram encontrados 2.060 assuntos cadastrados, desses foram selecionados os 20 com o maior número de publicações. A identificação destes 20 assuntos permitiu verificar quais temas estão sendo privilegiados pelos pesquisadores e instituições dedicados à produção de conhecimentos sobre saúde e desastres. Cada assunto foi cruzado com os outros quatro filtros escolhidos (País/Região como assunto, Idioma, Ano de publicação e Tipo de documento), o que permitiu uma análise relacional desta produção.

Após este procedimento, foi realizada nova seleção, utilizando o filtro Assunto principal.
Desta vez, foram selecionados todos os assuntos com mais de 100 publicações, com o objetivo de ampliar a compreensão acerca da diversidade de temas abordados na base. Foram encontrados 142 assuntos, que após análise foram agrupados em categorias ${ }^{6}$, tomando como referência a literatura científica sobre saúde e desastres. As buscas realizadas geraram um volume elevado de resultados. Por este motivo, foram agregados às discussões e apresentados da seguinte forma. Primeiro, foram incluídos os resultados referentes à toda base especializada, analisada a partir dos filtros País/Região, Idioma, Ano de publicação e Tipo de documento. Em seguida, os resultado e as discussões referentes aos 20 assuntos com maior número de publicações, confrontando com os mesmos filtros. Por último, apresentadas as dez categorias estabelecidas a partir dos 142 assuntos com mais de 100 publicações, seguidas das discussões referentes a cada uma delas.

\section{Resultados e discussão}

Do total de 19.170 publicações, por meio do filtro País/Região como assunto, foram encontrados os subcontinentes América do Sul (2.372) e América Central (2.210) com maior frequência de publicações. Em relação ao filtro Idioma, 49\% estava em espanhol e 47\% em inglês. Quanto ao tipo de documento, $73 \%$ das publicações estão no formato de monografia (relatórios técnicos, cartilhas, guias, manuais, entre outros). A década de 90 (Gráfico 1) apresentou maior número de publicações, com pico no ano 1993.

\section{Os 20 Assuntos da base "Desastres" com Maior Número de Publicações}

Na Tabela 1 foram sistematizados os resultados dos 20 assuntos principais com maior número de publicações, correlacionando-os com os filtros País/Região como assunto; Idioma, Tipo de documento e Ano com maior número de publicações.

Os três assuntos com maior número de publicações foram: Planejamento em desastres (3.184), Terremoto (2.598) e Prevenção e mitigação (2.553), nomes de países também apareceram como assuntos.

Ao aplicar o filtro País/Região como assunto, os subcontinentes América do Sul (1.833) e a América Central (1.688) apresentaram o maior número de publicações, equivalente ao resultado encontrado para toda a base. Para o filtro Idioma, dentre os 20 assuntos com maior número de pu- 


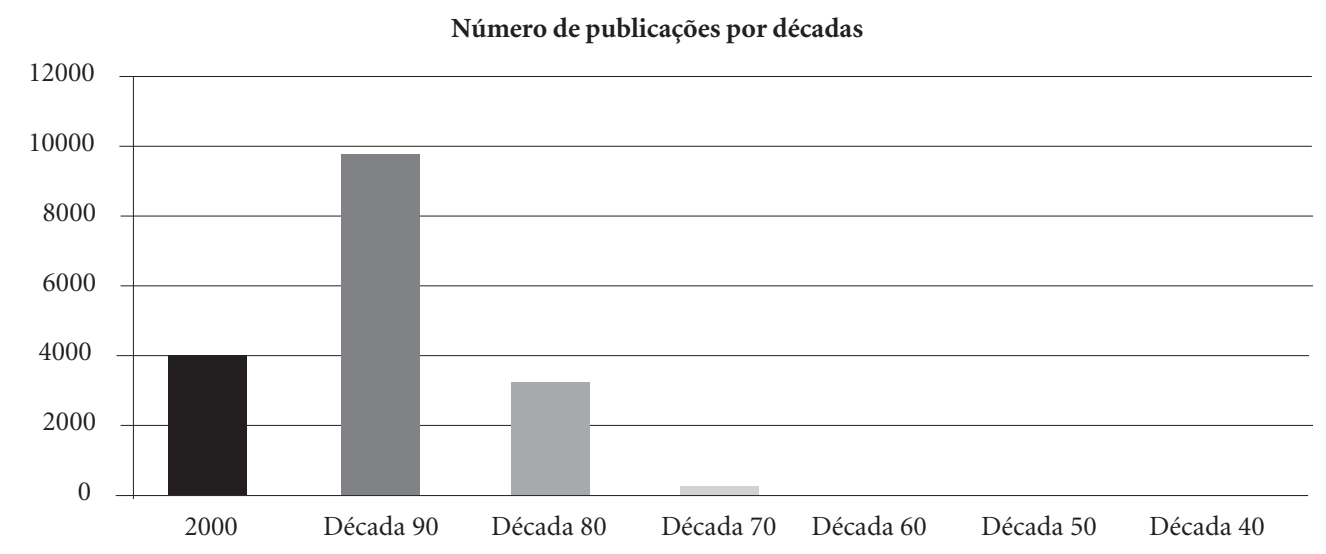

Gráfico 1. Décadas com maior número de publicações encontradas em todas as bases especializadas em desastres.

blicações, ocorre o predomínio do inglês (6.602) e espanhol (5.676), corroborando com os resultados encontrados em toda a base. Neste sentido, em relação aos 20 assuntos com maior número de publicações da base, poucos são os que apresentam o português como idioma. Os assuntos com maior número de publicações em português foram: Planejamento em desastres $(n=27)$, Prevenção e mitigação $(\mathrm{n}=16)$, Medidas de riscos $(\mathrm{n}=$ 16), Desastres $(n=13)$, Medidas de segurança ( $n$ =12), o que corresponde a menos de $1 \%$ do total de publicações. Os assuntos principais "Furacão, Engenharia, Estados Unidos, México, Erupções vulcânicas e Colômbia" não apresentam publicações em português.

Em relação ao filtro Tipo de documento, para os 20 assuntos, encontramos a Monografia com maior número de publicações (9.323), seguido de Congresso e Conferência (3.798) e Artigo (2.252), conforme encontrado em toda a base.

Para o filtro Ano de publicação, a década de 90 obteve também o maior número de publicações, com 2.152, ou seja, 85\%. Na década de 2000, merece destaque o assunto Mudança Climática, que em 2008 obteve 59 publicações.

Os resultados da Tabela 1 permitem uma primeira caracterização da base "Desastres" e discussão.

Ao analisar os 20 assuntos com maior número de publicação, foi encontrada uma variedade de temas como assunto principal, indicando que os descritores da base especializada são amplos e diversificados, permitindo um leque amplo de possibilidades de inclusão. Para discutir melhor estes 20 assuntos, agrupamos os mesmos por categorias que permitem dialogar com os 142 assuntos de maior destaque (Tabela 2), possibilitando uma discussão mais densa sobre os mesmos na base.

No primeiro agrupamento dos 20 assuntos com maior número de publicações aparecem em destaque os relacionados à categoria Processos e conceitos relacionados à redução de riscos (Planejamento em Desastres; Prevenção e Mitigação; Medição de Risco Avaliação de Danos; Medidas de Segurança; Participação Comunitária; Estratégias Nacionais). Dentre estes, predominam os relacionados ao tema da prevenção até a mitigação de danos, com foco no planejamento e estratégias nacionais envolvendo participação comunitária.

Dentre os 142 assuntos presentes na Tabela 2, esta categoria reuniu o maior número de assuntos. Analisando com maior detalhe, encontramos assuntos relacionados à ações técnicas e administrativas que envolvem diferentes setores (Planejamento em Desastres, Avaliação de Danos), etapas da gestão do risco (Prevenção e Mitigação, Reconstrução), bem como conceitos a serem apropriados por profissionais que trabalham com o tema desastres (Vulnerabilidade, Adaptação). Estratégias, acordos de cooperação e políticas de desenvolvimento em diferentes âmbitos também foram agregadas (Estratégias Nacionais, Cooperação Internacional). Por fim, foram agrupados assuntos relativos a formas de organização e ato- 
Tabela 1. Os 20 assuntos com maior número de publicações encontradas utilizando o filtro "Assunto principal" e as correlações com os filtros "País/Região como assunto"; "Idioma” e "Ano com maior número de publicações".

\begin{tabular}{|c|c|c|c|c|c|c|}
\hline Assunto principal & $\begin{array}{c}\text { N. de } \\
\text { publicações }\end{array}$ & \multicolumn{2}{|c|}{$\begin{array}{l}\text { País / Região } \\
\text { como assunto }\end{array}$} & $\begin{array}{c}\text { N.de } \\
\text { publicações }\end{array}$ & Idioma & $\begin{array}{c}\text { N. de } \\
\text { publicações }\end{array}$ \\
\hline Planejamento em Desastres & 3.184 & \multicolumn{2}{|c|}{ América central } & 399 & Inglês & 1.567 \\
\hline Terremotos & 2.598 & \multicolumn{2}{|c|}{ América do Sul } & 324 & Inglês & 1.707 \\
\hline Prevenção e Mitigação & 2.553 & \multicolumn{2}{|c|}{ América central } & 355 & Espanhol & 1.353 \\
\hline Medição de Risco & 1.399 & \multicolumn{2}{|c|}{ América Central } & 224 & Espanhol & 753 \\
\hline Desastres Naturais & 1.133 & \multicolumn{2}{|c|}{ América do Sul } & 143 & Espanhol & 564 \\
\hline Avaliação de Danos & 1.056 & \multicolumn{2}{|c|}{ América Central } & 201 & Inglês & 560 \\
\hline Desastres & 937 & \multicolumn{2}{|c|}{ América do Sul } & 105 & Espanhol & 504 \\
\hline Inundações & 783 & \multicolumn{2}{|c|}{ América do Sul } & 176 & Inglês & 398 \\
\hline Furacões & 740 & \multicolumn{2}{|c|}{ América Central } & 261 & Inglês & 410 \\
\hline Efeito de Desastres na Saúde & 739 & \multicolumn{2}{|c|}{ América do Sul } & 99 & Inglês & 458 \\
\hline Engenharia & 671 & \multicolumn{2}{|c|}{ América do Sul } & 67 & Inglês & 480 \\
\hline Mudança Climática & 660 & \multicolumn{2}{|c|}{ América do Sul } & 122 & Espanhol & 326 \\
\hline Estados Unidos & 654 & \multicolumn{2}{|c|}{ América do Norte } & 638 & Inglês & 613 \\
\hline Medidas de Segurança & 629 & \multicolumn{2}{|c|}{ América do Sul } & 59 & Espanhol & 367 \\
\hline México & 588 & \multicolumn{2}{|c|}{ México } & 585 & Espanhol & 495 \\
\hline Erupções Vulcânicas & 578 & \multicolumn{2}{|c|}{ América do Sul } & 139 & Espanhol & 342 \\
\hline Participação Comunitária & 564 & \multicolumn{2}{|c|}{ América Central } & 103 & Espanhol & 355 \\
\hline Educação & 532 & \multicolumn{2}{|c|}{ América do Sul } & 88 & Espanhol & 332 \\
\hline Estratégias Nacionais & 529 & \multicolumn{2}{|c|}{ América Central } & 145 & Espanhol & 319 \\
\hline Colômbia & 513 & \multicolumn{2}{|c|}{ América do Sul } & 511 & Espanhol & 470 \\
\hline Assunto principal & $\begin{array}{r}\text { Tipo } \\
\text { docume }\end{array}$ & & $\begin{array}{c}\text { N. de } \\
\text { publicações }\end{array}$ & $\begin{array}{r}\text { Ano com ma } \\
\text { de publ }\end{array}$ & $\begin{array}{l}\text { or número } \\
\text { ações }\end{array}$ & $\begin{array}{c}\text { N. de } \\
\text { publicações }\end{array}$ \\
\hline Planejamento em Desastres & Monog & & 2.214 & 19 & & 223 \\
\hline Terremotos & Monog & & 2.002 & 19 & & 308 \\
\hline Prevenção e Mitigação & Monog & & 1.988 & 19 & & 259 \\
\hline Medição de Risco & Monog & & 1.055 & 19 & & 199 \\
\hline Desastres Naturais & Monog & & 806 & 19 & & 183 \\
\hline Avaliação de Danos & Monog & & 692 & 19 & & 88 \\
\hline Desastres & Monog & fia & 684 & 19 & & 139 \\
\hline Inundações & Monog & & 555 & 19 & & 63 \\
\hline Furacões & Monog & fia & 436 & 19 & & 128 \\
\hline Efeito de Desastres na Saúde & Monog & & 380 & 198 & & 56 \\
\hline Engenharia & Monog & & 593 & 19 & & 161 \\
\hline Mudança Climática & Monog & & 585 & 20 & & 59 \\
\hline Estados Unidos & Monog & fia & 399 & 19 & & 64 \\
\hline Medidas de Segurança & Monog & & 467 & 19 & & 50 \\
\hline México & Monog & & 422 & 19 & & 60 \\
\hline Erupções Vulcânicas & Monog & & 358 & 19 & & 46 \\
\hline Participação Comunitária & Monog & & 425 & 19 & & 38 \\
\hline Educação & Monog & & 423 & 19 & & 56 \\
\hline Estratégias Nacionais & Monog & & 380 & 19 & & 87 \\
\hline Colômbia & Monog & afia & 371 & 198 & & 46 \\
\hline
\end{tabular}

res chaves, para lidar com os problemas relativos aos desastres (Participação Comunitária, Organização e Defesa Civil). Nota-se na Tabela 2 que os assuntos com maior número de publicações tratam de ações técnico- administrativas e a gestão de risco de desastres.
Deve se considerar positivo a presença de um grande número de assuntos nesta categoria, uma vez que no marco das Funções Essenciais da Saúde Pública da OPAS 7 , o desenvolvimento de políticas, o planejamento e a realização de ações de prevenção, mitigação, preparação, resposta e re- 
Tabela 2. Categorias estabelecidas a partir da identificação dos 142 assuntos com maior número de publicações e os respectivos assuntos incluídos em cada uma.

\begin{tabular}{|c|c|c|}
\hline Categoria & Assunto Principal & N. de publicações \\
\hline Processos e conceitos relacionados & Planejamento em Desastres & 3.184 \\
\hline \multirow[t]{40}{*}{ à redução de riscos } & Prevenção e Mitigação & 2.553 \\
\hline & Medição de Risco & 1.399 \\
\hline & Avaliação de Danos & 1.056 \\
\hline & Medidas de Segurança & 629 \\
\hline & Participação Comunitária & 564 \\
\hline & Estratégias Nacionais & 529 \\
\hline & Cooperação Internacional & 509 \\
\hline & Avaliação & 463 \\
\hline & Organização e Administração & 398 \\
\hline & Reconstrução & 374 \\
\hline & Análise de Vulnerabilidade & 373 \\
\hline & Defesa Civil & 340 \\
\hline & Estratégias Locais & 318 \\
\hline & Fatores Socioeconômicos & 301 \\
\hline & Recuperação Pós-Desastres & 289 \\
\hline & Prevenção de Acidentes & 288 \\
\hline & Sistemas de Informação & 275 \\
\hline & Administração de Desastres & 266 \\
\hline & Cooperação Técnica & 255 \\
\hline & Estratégias Regionais & 196 \\
\hline & Cruz Vermelha & 193 \\
\hline & Controle de Risco & 191 \\
\hline & Evacuação & 170 \\
\hline & Estratégias & 164 \\
\hline & Busca e Resgate & 149 \\
\hline & Vulnerabilidade & 148 \\
\hline & Adaptação & 146 \\
\hline & Fatores de Risco & 139 \\
\hline & Planejamento de Cidades & 139 \\
\hline & Reabilitação & 138 \\
\hline & Planos de Emergência & 138 \\
\hline & Simulação & 133 \\
\hline & Alerta em Desastres & 129 \\
\hline & Risco & 128 \\
\hline & Métodos & 127 \\
\hline & Desenvolvimento Econômico & 125 \\
\hline & Equipamentos e Provisões & 115 \\
\hline & Triagem & 110 \\
\hline & Organização Comunitária & 109 \\
\hline & Assistência Internacional em Desastres & 108 \\
\hline
\end{tabular}

abilitação são consideradas vitais para reduzir o impacto dos desastres sobre a saúde pública. Porém, para uma melhor organização, seria importante que cada um dos assuntos integrasse um conjunto de categorias específicas e relacionadas aos processos de redução de riscos de desastres, tais como: prevenção de riscos; preparação e respostas; respostas e reabilitação; recuperação e reconstrução ${ }^{8}$.
No segundo agrupamento temos a categoria Países. Embora para o filtro País/Região como assunto, os subcontinentes América do Sul e América Central tenham apresentado maior número de publicações, dentre os 20 assuntos principais com maior número de publicações (Tabela 1) aparecem países como Estados Unidos e México, situados na América do Norte nos primeiros lugares, para, só depois, aparecer Colômbia. 
Tabela 2. continuação

\begin{tabular}{|c|c|c|}
\hline Categoria & Assunto Principal & N. de publicações \\
\hline \multirow{17}{*}{$\begin{array}{l}\text { Processos/ ações relacionados } \\
\text { especificamente com a saúde }\end{array}$} & Emergências em Desastres & 445 \\
\hline & Serviços Médicos de Emergência & 396 \\
\hline & Planejamento Hospitalar & 339 \\
\hline & Cuidados Médicos & 231 \\
\hline & Políticas Públicas de Saúde & 224 \\
\hline & Hospitais & 221 \\
\hline & Saúde Pública & 214 \\
\hline & Serviços de Saúde & 210 \\
\hline & Assistência a Feridos em Massa & 192 \\
\hline & Socorro em Desastres & 164 \\
\hline & Saúde Ambiental & 163 \\
\hline & Epidemiologia & 146 \\
\hline & Primeiros Socorros & 126 \\
\hline & Vigilância Epidemiológica & 123 \\
\hline & Planejamento em Saúde & 119 \\
\hline & Atenção à Saúde & 116 \\
\hline & Medicina de emergência & 102 \\
\hline \multirow{3}{*}{$\begin{array}{l}\text { Processos/ ações relacionados a água } \\
\text { e saneamento }\end{array}$} & Abastecimento de água & 235 \\
\hline & Saneamento em desastres & 170 \\
\hline & Água potável & 149 \\
\hline \multirow[t]{6}{*}{ Impactos/ efeitos sobre à saúde } & Efeitos de desastres na saúde & 739 \\
\hline & Saúde Mental & 328 \\
\hline & Impacto de Calamidades & 322 \\
\hline & Impactos na saúde & 148 \\
\hline & Influenza Humana & 113 \\
\hline & Efeitos de Radiação & 107 \\
\hline \multirow[t]{19}{*}{ Tipo de desastres } & Terremoto & 2.598 \\
\hline & Desastres naturais & 1.133 \\
\hline & Desastres & 937 \\
\hline & Inundação & 783 \\
\hline & Furacões & 740 \\
\hline & Erupção vulcânica & 578 \\
\hline & Substância perigosa & 473 \\
\hline & Sismos & 451 \\
\hline & Acidentes químicos & 413 \\
\hline & Deslizamentos de terra & 329 \\
\hline & Incêndio & 248 \\
\hline & Guerras & 222 \\
\hline & Desastres provocados pelo homem & 212 \\
\hline & Tsunamis & 211 \\
\hline & Secas & 188 \\
\hline & Ameaças & 158 \\
\hline & Desastre industrial & 142 \\
\hline & Liberação nociva de radioativos & 137 \\
\hline & Acidentes aeronáuticos & 106 \\
\hline
\end{tabular}

continua

Analisando a produção disponível na base para estes três países, que aparecem em destaque nos 20 principais assuntos, encontramos diferen- ças importantes. Para os Estados Unidos, temas como terremotos e abalos sísmicos, tornados e furacões, inundações, acidentes químicos e pro- 
Tabela 2. continuação

\begin{tabular}{|c|c|c|}
\hline Categoria & Assunto Principal & N. de publicações \\
\hline \multirow[t]{23}{*}{ Países } & Estados Unidos & 654 \\
\hline & México & 588 \\
\hline & Colômbia & 513 \\
\hline & Costa Rica & 477 \\
\hline & Peru & 472 \\
\hline & América Central & 318 \\
\hline & El Salvador & 303 \\
\hline & Guatemala & 302 \\
\hline & Nicarágua & 275 \\
\hline & América Latina & 268 \\
\hline & Japão & 213 \\
\hline & Equador & 208 \\
\hline & Honduras & 191 \\
\hline & Região do Caribe & 180 \\
\hline & Venezuela & 173 \\
\hline & Brasil & 157 \\
\hline & Espanha & 142 \\
\hline & Chile & 134 \\
\hline & Panamá & 126 \\
\hline & Países em Desenvolvimento & 124 \\
\hline & Austrália & 121 \\
\hline & Canadá & 114 \\
\hline & Bolívia & 101 \\
\hline \multirow[t]{6}{*}{ Temáticas ambientais } & Mudança climática & 660 \\
\hline & Meio ambiente & 294 \\
\hline & Impacto ambiental & 121 \\
\hline & Poluição ambiental & 111 \\
\hline & Desenvolvimento sustentável & 104 \\
\hline & Fenômeno El Niño & 101 \\
\hline \multirow{9}{*}{$\begin{array}{l}\text { Pesquisa, educação e disseminação } \\
\text { de conhecimento }\end{array}$} & Educação & 532 \\
\hline & Educação em Desastres & 354 \\
\hline & Instituições acadêmicas & 344 \\
\hline & Materiais de ensino & 344 \\
\hline & Manuais & 229 \\
\hline & Pesquisa & 185 \\
\hline & Meios de comunicação & 154 \\
\hline & Telecomunicações & 118 \\
\hline & Serviços de Informação & 114 \\
\hline \multirow[t]{6}{*}{ Grupos vulneráveis } & Refugiados & 229 \\
\hline & Crianças & 207 \\
\hline & Pessoas deslocadas & 195 \\
\hline & Mulheres & 160 \\
\hline & Grupos de risco & 132 \\
\hline & Vítimas de desastres & 109 \\
\hline
\end{tabular}

continua

dutos perigosos, gestão do risco de desastres e engenharia costumam ser os de maior recorrência. Para o México há uma variedade maior entre os temas, bem como maior número de publicações relacionadas à vulnerabilidade e impacto social dos desastres e sistema da defesa civil. $\mathrm{Na} \mathrm{Co}-$ 


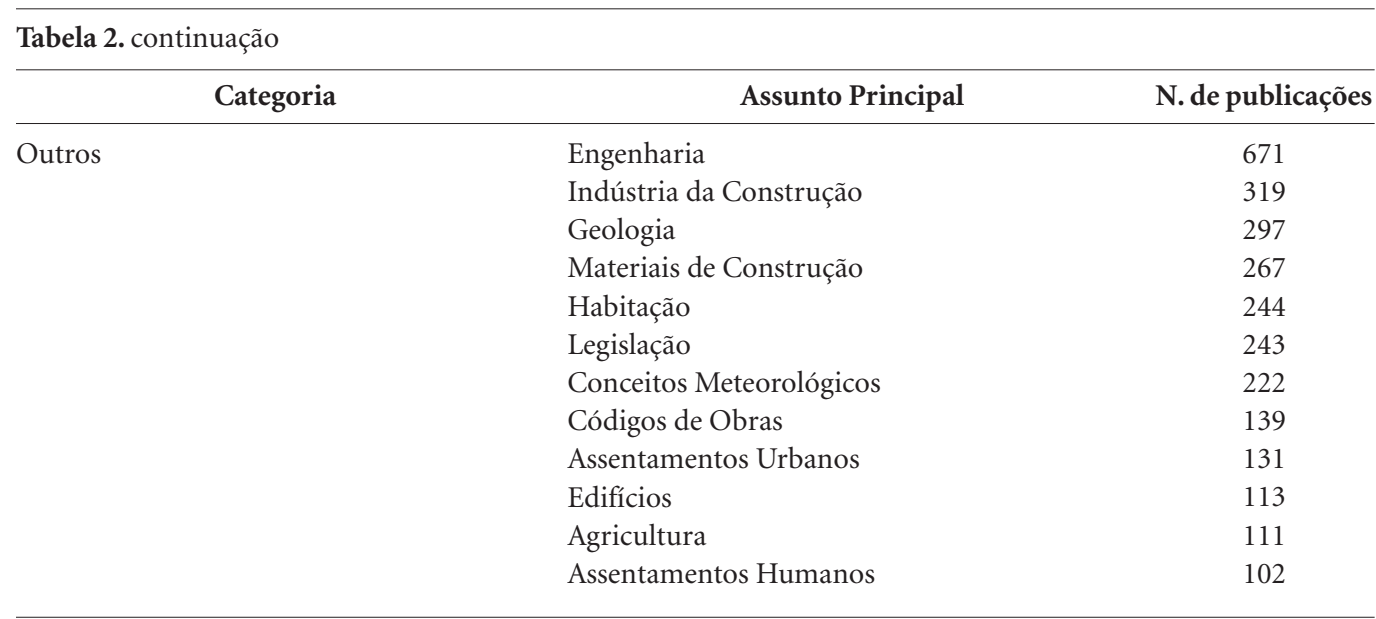

lômbia, assim como no México, foi encontrado um maior número de publicações relacionadas ao tema vulnerabilidade, enquanto nos Estados Unidos, maior número de publicações relacionadas aos impactos econômicos dos desastres.

Porém, ainda na Tabela 1, quando correlacionamos os assuntos principais com País/Região, constatamos que América do Sul e América Central, junto com México, aparecem em 19 dos 20 assuntos. Esta predominância de países da AL\&C também aparece na Tabela 2, uma vez que a maioria dos 23 países que aparecem se situam nestes subcontinentes, o que pode ser explicado por dois aspectos que se inter-relacionam. Primeiro, uma tendência geral da base, uma vez que a BVS reúne publicações principalmente da AL\&C. Segundo, pelo fato do continente americano ser o segundo mais afetado em número de desastres e fatalidades e o primeiro em impacto, conforme dados do $\mathrm{CRED}^{5}$. Além disso, AL\&C são acometidas tanto por desastres intensivos como terremotos, furacões, erupções vulcânicas, inundações bruscas, quanto por desastres extensivos, como as inundações graduais e secas ${ }^{9}$.

Ainda na Tabela 2, constatamos que o Brasil ocupou o décimo sexto lugar dos países como assunto principal, com 157 publicações. Com relação aos diferentes tipos de desastres, as inundações são o foco da maioria das publicações. Há poucos documentos relacionados aos acidentes químicos e demais desastres provocados pelo homem, pouquíssimos sobre incêndios e secas e nenhum especificamente sobre deslizamentos de terra no Brasil. Por outro lado, chama a atenção o grande número de publicações relacionadas ao tema defesa civil. Foram encontradas poucas pu- blicações relacionadas ao tema vulnerabilidade social (como nos casos da Colômbia e México) e, menos ainda, sobre os impactos econômicos (como nos Estados Unidos) dos desastres no país.

A categoria Países revelou que Brasil, como assunto principal nos estudos sobre desastres, teve reduzido volume de publicação em comparação com os demais países da AL\&C. Considerando a extensão territorial, a constante ocorrência de desastres e a existência de uma comunidade científica robusta no país, era de se esperar uma participação mais expressiva. Mesmo países com extensão territorial muito menor, como o México, El Salvador e Costa Rica, possuem um número de publicações superior.

Em relação ao idioma, que em muitos casos expressa a importância dos países ou regiões na produção, constatamos na Tabela 1 que, para $12(60 \%)$ dos 20 assuntos, o idioma com maior número de publicações foi o espanhol, sendo seguido pelo inglês. Quando analisamos a presença do idioma português para cada um dos 20 assuntos, este não ultrapassou 1\%. Esta situação fica desfavorável para os idiomas espanhol e português, quando tomamos como referência a base Medline, que hoje também integra o portal "BVS Preparação e resposta aos Desastres”, e tem como foco a literatura científica biomédica (revistas científicas e livros online). Na base "Desastres" o documento mais antigo data de 1942, e a busca realizada para este artigo foi concluída em 24 de setembro de 2013. Ao realizar uma busca no $\mathrm{Me}$ dline, cobrindo o período de 1 de janeiro de 1942 (ano da primeira publicação na "Desastres") a 31 de setembro de 2013 (mês e ano da busca realizada para este artigo), encontramos 12.474 pu- 
blicações que tiveram o termo desastre (disaster) no título ou resumo. Do total destas publicações, $85 \%$ estão em inglês, $0,5 \%$ em espanhol e $0,01 \%$ em português, o que é o reflexo da pequena produção que os países de lingua portuguesa e espanhola, entre estes os da AL\&C, possuem em uma base que tem como foco revistas científicas e livros.

Especificamente para o caso do Brasil, quando comparados aos indicadores bibliométricos do Scielo Saúde Pública ${ }^{10}$, que engloba revistas da AL\&C, constata-se que do total de 19.242 artigos publicados entre 2000 e 2012, 40\% tiveram o Brasil como país de afiliação do autor. Segundo relatório sobre os atuais desafios e tendências da inovação, o Brasil situa-se em $14^{\circ}$ lugar no ranking mundial da produção científica, sua participação está em crescimento, sendo o país líder no da produção científica da América Latina ${ }^{11}$. O Brasil vem avançando bastante no âmbito da Saúde Pública e possui produção expressiva na AL\&C, mas estes avanços e expressão ainda não chegaram ao tema dos desastres, como indicam os dados da base "Desastres" e, de modo mais drástico, no Medline.

No terceiro agrupamento temos Tipo de desastres. Na Tabela 1 assuntos como Desastres e Desastres Naturais combinam-se com tipos de desastres de origem geológica, como Terremotos e Erupções Vulcânicas; de origem hidrológica, como Inundações; de origem meteorológica, como Furacões; sendo que estes dois últimos muito relacionados ao assunto da Mudança Climática, que integra a categoria Temáticas ambientais que será analisada posteriormente.

Na Tabela 2, na categoria Tipo de desastres estão incluídos os assuntos relacionados aos desastres naturais e tecnológicos, sendo 16 tipos específicos de desastres. Nove desses 16 são ameaças naturais, cujo volume de publicações corresponde a $78 \%$ desta categoria. Os outros sete assuntos tratam de desastres tecnológicos, correspondendo a $22 \%$ desta categoria.

Para melhor interpretar os dados desta categoria, os tipos de desastres foram cruzados com os filtros Ano de publicação, País ou região, Idioma e Tipo de documento mais frequente, os resultados estão disponíveis na Tabela 3.

$\mathrm{O}$ assunto Terremoto teve o maior número de publicações em 1993, principalmente na América do Sul; Inundação teve o maior número de publicações em 1995, também na América do Sul; Furacões, teve o maior número de publicações em 1999, principalmente na América Central; todos tiveram o inglês como idioma predominante.
A América do Sul e a América Central foram as principais regiões relacionadas com os diferentes tipos de desastres, vindo em seguida a América do Norte. Em relação ao idioma temos o predomínio do inglês.

Para todos os tipos de desastres como assunto, o documento mais encontrado foi a monografia, exceto para Acidentes Aeronáuticos, cujo documento mais frequente foi artigo científico.

A categoria Tipo de desastres revelou maior concentração dos estudos nos desastres naturais do que nos tecnológicos. Na análise ficou evidente que, dentre os desastres naturais, os intensivos despertam maior interesse e investimentos por parte dos pesquisadores e instituições, uma vez que terremotos, furacões e erupção vulcânica estão entre os quatro tipos de desastres com o maior número de publicações (Tabela 1).

Ainda na Tabela 1, temos alguns assuntos que apareceram isolados, não permitindo agrupamentos, tais como Efeitos de Desastres na Saúde, Educação e Mudança Climática. Considerandose a grande importâmcia de ambos, analisamos os mesmos a partir das categorias que integram na Tabela 2.

Incluindo especificamente o termo saúde, encontra-se somente o assunto Efeitos de Desastres na Saúde, o qual na Tabela 2 integra a categoria Impactos/efeitos sobre à saúde. Nesta categoria estão incluídos os assuntos relacionados aos impactos e efeitos sobre a saúde das populações perante os desastres. Abrange desde assuntos mais amplos, como os Efeitos de Desastres na Saúde, Impacto de Calamidades e Impactos na Saúde até assuntos mais específicos como Saúde Mental, Influenza Humana e os Efeitos da Radiação.

Ao comparar estes assuntos aos principais impactos dos desastres naturais na saúde presentes na literatura ${ }^{9,12,13}$, não foram encontrados efeitos ou impactos comuns a vários tipos de desastres naturais como as doenças infecciosas e parasitárias causadas por vetores e hospedeiros, bem como doenças respiratórias e circulatórias, lesões e causas externas.

Outras duas categorias na Tabela 2 se encontram diretamente relacionadas com o tema saúde, sendo estas a de Processos/ações relacionados especificamente com a saúde e Processos/ações relacionados à agua e saneamento.

Na primeira categoria foram agrupados os assuntos ligados diretamente aos processos e ações que envolvem a organização do Setor Saúde para o enfrentamento dos desastres. Nota-se que a maioria dos assuntos agrupados nessa categoria se refere diretamente aos processo de preparação 
Tabela 3. Assuntos incluídos na categoria Tipo de desastres, encontrados dentre os 142 assuntos principais e correlações com os filtros "País e Região como assunto", "Idioma” e "Ano com maior número de publicações".

\begin{tabular}{|c|c|c|c|c|c|c|c|}
\hline $\begin{array}{c}\text { Assuntos incluídos na categoria } \\
\text { Tipo de desastres }\end{array}$ & $\begin{array}{c}\text { N. de } \\
\text { publicações }\end{array}$ & País / Região & $\begin{array}{c}\text { N. de } \\
\text { publicações }\end{array}$ & Idioma & $\begin{array}{c}\text { N. de } \\
\text { publicações }\end{array}$ & $\begin{array}{c}\text { Ano com } \\
\text { maior número } \\
\text { de publicações }\end{array}$ & $\begin{array}{c}\text { N. de } \\
\text { publicações }\end{array}$ \\
\hline Terremoto & 2.598 & América do Sul & 324 & Inglês & 1.707 & 1993 & 308 \\
\hline Inundação & 783 & América do Sul & 176 & Inglês & 398 & 1995 & 63 \\
\hline Furacões & 740 & América Central & 261 & Inglês & 410 & 1999 & 128 \\
\hline Erupção Vulcânica & 578 & América do Sul & 139 & Espanhol & 342 & 1993 & 46 \\
\hline Substância Perigosa & 473 & América do Norte & 49 & Inglês & 282 & 1988 & 43 \\
\hline Sismos & 451 & América do Sul & 102 & Espanhol & 315 & 1997 & 59 \\
\hline Acidentes Químicos & 413 & América do Norte & 32 & Inglês & 276 & 1993 & 55 \\
\hline Deslizamentos de terra & 329 & América Central & 126 & Espanhol & 205 & 2003 & 41 \\
\hline Incêndio & 248 & América do Norte & 22 & Espanhol & 130 & 1992 & 18 \\
\hline Guerra & 222 & Ásia & 24 & Inglês & 151 & 1993 & 24 \\
\hline Desastres Provocados pelo Homem & 212 & México & 18 & Inglês & 117 & 1991 & 24 \\
\hline Tsunamis & 211 & América do Sul & 57 & Inglês & 110 & 2010 & 28 \\
\hline Secas & 188 & África & 38 & Espanhol & 98 & 1992 & 13 \\
\hline Desastre Industrial & 142 & América do Norte & 12 & Inglês & 95 & 1993 & 27 \\
\hline Liberação Nociva de Radioativos & 137 & América do Sul & 13 & Inglês & 105 & 1986 & 36 \\
\hline Acidentes Aeronáuticos & 106 & América do Norte & 20 & Inglês & 72 & 1980 & 1111 \\
\hline Total & 7.841 & & & & & & \\
\hline
\end{tabular}

e resposta (envolvendo exemplos como Planejamento Hospitalar; Emergências em Desastres; Serviços de Saúde; Cuidados Médicos; Assistência e Socorro) com foco nas respostas imediatas, restando aos processos de recuperação, reabilitação e reconstrução da saúde no período pós-desastre um número menor de publicações.

$\mathrm{Na}$ segunda categoria foram agrupados os assuntos Abastecimento de Água, Saneamento em Desastres e Água Potável, que agregam temas referentes aos processos e ações relacionados à gestão da água e saneamento em situações de desastre. Englobam desde ações de abastecimento e monitoramento da qualidade da água para a população afetada, incluindo situações de abrigos e de refugiados, até medidas de avaliação e formas alternativas de tratamento da água. Também incluem processos e ações de saneamento em situação de desastre, como construção de sistemas, tratamento e disposição final de excretas humanas e animais e de resíduos sólidos, assim como medidas de educação para promover a higiene e o funcionamento dos sistemas de esgotamento sanitário nas comunidades afetadas, abrigos e campos de refugiados.

$\mathrm{O}$ acesso e a qualidade da água, bem como o acúmulo de resíduos sólidos são situações de risco muito comuns após desastres e que criam con- dições para o aumento das doenças relacionadas aos vetores e hospedeiros após estes eventos, quadro recorrente na AL\&C, de modo que devem ser consideradas tanto pelos gestores como pela comunidade científica do setor saúde, não somente nos processos de preparação e respostas, como nos processos pós-desastres de reabilitação, recuperação e reconstrução ${ }^{13,14}$.

$\mathrm{O}$ assunto Educação foi outro que apareceu isolado na Tabela 1. Considerando-se sua importância como um dos determinantes sociais da saúde, bem como uma das prioridades de ação no Marco de Ação de Hyogo (documento de referência das Nações Unidas para a Redução de Riscos de Desastres no nível global para o período 2005-2015) $)^{15}$ para construção de uma cultura de segurança e resiliência em todos os níveis, analisamos o mesmo a partir da sua inserção na categoria Pesquisa, educação e disseminação do conhecimento na Tabela 2.

Foram incluídos nesta categoria os assuntos relacionados à pesquisa, educação, ensino, informação e comunicação. Estes assuntos fomentam as ações de redução de riscos de desastres e demonstram a preocupação em gerar conhecimento e disseminá-lo em diferentes âmbitos. Dentre os assuntos encontrados, Educação e Educação em Desastres, Instituições Acadêmicas e Mate- 
riais de Ensino foram os que apresentaram maior número de publicações. Assuntos relacionados à produção de conteúdos práticos como Manuais, bem como sua disseminação através de Serviços de Informação, Meios de Comunicação e Telecomunicações também tiveram destaque.

Os assuntos agregados a esta categoria fomentam ações para a redução de risco de desastres, pois a produção de conhecimento e sua difusão contribuem para dispor dados e informações importantes para propor estratégias, planos e políticas, elaborar leis, guias, manuais, construir sistemas de informação e de divulgação desta, para tomada de decisão, encontrar formas mais adequadas de comunicação de risco, formar recursos humanos em diferentes níveis, criar metodologia de educação para população em risco e fomentar a pesquisa sobre saúde e desastres ${ }^{8}$. Aplicações práticas de conhecimetos das ciências naturais e sociais são essenciais para reduzir as vulnerabilidades. Deste modo, informações, bem como o desenvolvimento de capacidades científicas e técnicas relevantes, especialmente em países em desenvolvimento, são fundamentais ${ }^{16}$. Avaliações de risco e acompanhamento de programas de educação pública têm contribuído para os altos níveis de consciência e de preparação da população. Considerando que a prevenção a desastres e o desenvolvimento de resiliência social são igualmente dependentes do conhecimento científico e técnico produzido e da disseminação do mesmo acoplados à programas de desenvolvimento da inovação e da educação, o assunto educação e todos os outros desta categoria são fundamentais para atuar sobre os determinantes sociais dos desastres e construir uma cultura de segurança e resiliência em todos os níveis.

Mudança Climática foi o terceiro assunto que apareceu isoladamente na Tabela 1. A associação dos desastres com as mudanças climáticas e seus impactos na saúde podem ter resultado num maior interesse dos pesquisadores em produzir estudos e publicações a partir desta relação, principalmente nos países da AL\&C. A região historicamente sofre com eventos meteorológicos (ciclones tropicais e extra-tropicais, furacões, tornados e vendavais), hidrológicos (alagamentos, enchentes, inundações graduais e bruscas) e climatológicos (estiagem e seca, queimadas e incêndios florestais, chuvas de granizo, geadas e ondas de frio e de calor), que se encontram direta e indiretamente relacionados às mudanças climáticas.

Ao mesmo tempo, o assunto da mudança climática se insere diretamente na categoria das $T e$ máticas ambientais, presentes na Tabela 2. Nesta categoria encontramos pares de assuntos muito próximos, como Mudança Climática e o Fenômeno El Niño, Impacto Ambiental e Poluição Ambiental, Meio Ambinte e Desenvolvimento Sustentável.

A análise permitiu evidenciar alguns momentos em que estas temáticas ambientais foram debatidas na sociedade e, consequentemente, mereceram atenção especial por parte da comunidade acadêmica.

O assunto Mudança Climática predomina no ano de 2008, um ano após o lançamento do Quarto Relatório em 2007, alertando para os impactos futuros e aumento da frequência e intensidade de eventos como ondas de calor e chuvas torrenciais, secas, ciclones tropicais e marés altas elevadas ${ }^{17}$. Ainda no ano de 2007, o grupo de cientistas que trabalhou no mesmo ganhou o prêmio Nobel da Paz.

O assunto Poluição Ambiental teve seu auge em 1989, fim da década de 80 quando foi publicado o Relatório Nosso Futuro Comum trazendo a tona a questão ambiental como nunca antes visto $^{18}$. Meio Ambiente e Impactos Ambientais predominaram na década de 1990, provavelmente pelo reflexo da Rio 92, tendo em vista a expectativa criada pela Agenda $21^{19}$. O assunto Fenômeno El Niño tem no ano de 2000 o maior número de públicações, sendo que os anos de 1997-1998 foram anos de ocorrência deste evento com forte intensidade, resultando em inundações e secas mais intensas.

Encontrar temáticas ambientais na base "Desastres" é importante, pois a redução do risco de desastres está diretamente relacionada à redução de impactos ambientais, que intensificam as ameaça naturais capazes de desencadear o desastre. No Marco de Ação Hyogo a integração da Redução de Riscos de Desastres (RRD) nas políticas e planejamento do desenvolvimento sustentável é uma prioridade, tanto que constituiu um dos sete temas prioritários para a Rio $+20^{20}$.

Neste sentido, há necessidade contínua de investimentos em pesquisa básica e aplicada que promova desenvolvimento sustentável ${ }^{16}$. Entretanto, no Brasil, revisões da literatura científica sobre saúde e ambiente ${ }^{21,22}$, cobrindo um período de 1992 à 2003, não identificaram o tema desastres entre os impactos ambientais de maior destaque, o que torna urgente investimentos para a pesquisa sobre este tema no Brasil, como vem ocorrendo em outras partes do mundo ${ }^{23}$.

Interessante observar que, se o conceito de desastres envolve a combinação de ameaças naturais (terremotos, furacões, inundações ou secas, 
por exemplo) e condições de vulnerabilidade (o que envolve as condições sociais e econômicas diretamente relacionadas aos determinantes sociais da saúde $)^{24}$, tanto o assunto vulnerabilidade ou os assuntos relacionados aos grupos vulneráveis, não tenham aparecido na Tabela 1 entre os 20 assuntos com maior número de públicações. $\mathrm{O}$ foco predominante é ainda nas ameaças naturais (terremotos, erupções vulcânicas e inundações aparecem) com base nas Ciências Naturais, com bem menor destaque para o foco centrado nas pessoas e nas Ciências Sociais e da Saúde.

$\mathrm{Na}$ Tabela 2, uma das categorias que surgiu a partir do levantamento dos 142 assuntos com maior número de publicações foi Grupos vulneráveis, envolvendo os assuntos relacionados ao conjunto da população que sofre os desastres (Refugiados, Pessoas Deslocadas e Vítimas de Desastres), bem como aos grupos específicos, tais como Crianças, Mulheres e Grupos de Risco.

Esta categoria revelou um maior volume de investigações acerca de grupos socialmente vulneráveis e inclui mulheres e crianças que, segundo dados para o tema na Rio $+20^{25}$, possuem 14 vezes mais chances de óbito em um desastre. Notamse poucos trabalhos contidos na base "Desastres" que investigam especificamente a vulnerabilidade da população idosa e pessoas com deficiências. Segundo a literatura, crianças, gestantes e idosos, principalmente das zonas rurais, são considerados os grupos mais vulneráveis aos efeitos da seca a curto e longo prazos, por exemplo ${ }^{12}$. Já nas enchentes, os grupos vulneráveis são crianças, gestantes, idosos e pessoas com deficiência ${ }^{13}$. Mesmo sendo os idosos o grupo vulnerável comum a ambos tipos de desastres, estes não aparecem dentre os 142 assuntos com o maior numero de publicações da base "Desastres". No entanto, encontramos os assuntos "direito dos idosos" e "assistência a idosos", que ocupam lugar bem inferior (877 e $1939^{\circ}$ respectivamente), dentre os 2.060 encontrados por meio do filtro Assunto principal da base "Desastres", indicando que existem poucos trabalhos disponibilizados que tratem especificamente da população idosa. Ou ainda, que este assunto possa estar organizado de uma forma que seu acesso na base seja dificultado.

Por fim, na Tabela 2, organizamos na categoria Outros os que não encontravam nenhuma correspondência direta com as anteriores e que consideramos prioritárias. São assuntos diretamente relacionados à construção de obras de engenharia (Engenharia, Indústria da Construção, Materiais de Construção, Códigos de Obras), moradias e organização do espaço (Habitação,
Edifícios, Assentamentos Urbanos, Assentamentos Humanos), ou a dinâmica da terra (Geologia e Meteorologia).

Entre todos os assuntos com maior número de publicações presentes nas Tabelas 1 e 2, a monografia também foi o tipo de documento mais encontrado, reafirmando que na base "Desastres" relatórios técnicos, cartilhas, guias e manuais, entre outros, são abordados principalmente através de publicações voltadas mais diretamente para os profissionais que atuam nos desastres e gestores.

De modo geral, o presente artigo chama a atenção para a importância de se analisar o que as bases e suas redes oferecem e identificar o potencial da informação disponível. Em trabalho exploratório semelhante, Packer et al. ${ }^{26}$ analisam a distribuição da produção científica regional, nacional e internacional na área de informação e comunicação em saúde. Estudos desta natureza reafirmam que atualmente há um enorme arcabouço de produção científica disponível em diferentes bases, que, no entanto, não significa necessariamente um maior e melhor acesso no meio a tanta informação. A simples comparação entre o tipo de documento, países/regiões e idiomas (privilegiados na base "Desastres" e na base Medline, ambas integrando hoje o novo portal BVS "Preparação e resposta aos desastres"), já demonstra isto, com a primeira apresentando grande potencial para o público de técnicos e gestores, e a segunda para o público de pesquisadores e pós-graduandos.

\section{Considerações finais}

Apesar dos importantes ganhos para o continente americano com a criação da BVS e depois da base "Desastres", há ainda alguns desafios a serem enfrentados, particularmente por países como Brasil.

O primeiro é não só aumentar a produção de conhecimentos e informações sobre a redução de desastres e saúde em português, mas também agregar e compartilhar a mesma na BVS. Como demonstrado, ter menos de $1 \%$ das publicações na base "Desastres" não corresponde ao lugar que o Brasil ocupa na produção científica global, ou mesmo do continente americano no Scielo Saúde Pública.

O segundo é que o aumento e compartilhamento da produção técnico-científica em português responda não só aos desafios dos desastres intensivos mais correntes no Brasil, como inundações bruscas e deslizamentos, mas também dos desastres extensivos, particularmente a seca. A 
seca é o tipo de desastre mais frequente e ao mesmo tempo crônico no Brasil, carecendo de uma produção técnica-científica que responda aos desafios que a mesma provoca para a saúde coletiva.

O terceiro é não só aumentar a produção técnico-científica sobre desastres e saúde, como, no caso da seca, exemplificado no parágrafo anterior, mas também que englobe dois aspectos importantes: 1) Investimentos em estudos sobre a atuação do setor saúde nas etapas de Redução do Risco, ampliando o escopo para além das respostas imediatas focadas nas urgências e emergências, incluindo tanto as de prevenção, como de reabilitação, recuperação e reconstrução; 2) Investimentos em estudos que ampliem a compreensão dos impactos e efeitos sobre a saúde da população exposta aos desastres, não só para os imediatos, mas também os de médio e longo prazos, considerando os diferentes grupos vulneráveis.

Para que isto possa ser realizado e contribuir para fortalecer a agregação, compartilhamento e acesso do conhecimento e informações sobre desastres na e para a AL\&C, é necessário que a base "Desastres" seja continuamente atualizada e tenha como foco as monografias, já que nas outras bases como Medline encontra-se a literatura científica presente nos periódicos e livros. Ao mesmo tempo é necessário que a construção de ferramentas adequadas para tornar as buscas mais eficientes para os diferentes públicos, como gestores, pesquisadores, profissionais de serviços (saúde e defesa civil, por exemplo) e o público de modo geral, seja um processo contínuo que com- bine a criatividade humana com ajuda da tecnologia, tendo como base conceitos relacionados aos processos de gestão para a redução de riscos de desastres em todos os seus aspectos ${ }^{8}$.

Nos últimos anos, a necessidade de compartilhar informações (não apenas científicas, mas também técnicas) sobre o tema desastres ganhou tanta importância no cenário internacional, que a BVS desenvolveu o portal "Preparação e resposta a desastres", o qual inclui, entre outras, a base "Desastres". A intenção é que o portal possa tornar ainda mais acessível a produção científica, material técnico, guias práticos e planos de preparação, bem como informações ao público e facilitar o estabelecimento de redes sociais, ampliando a proposta e as possibilidades da base "Desastres".

Se por um lado o idioma inglês é universal e está ao alcance da maioria dos pesquisadores, inclusive no Brasil, para muitos gestores, profissionais de saúde e defesa civil, além da população em geral, é ainda uma barreira no acesso à informação. Neste sentido, a disponibilização de conteúdo científico e técnico principalmente nos idiomas português e espanhol, facilitaria o intercâmbio entre a academia, as empresas, os serviços públicos e a sociedade. Nossa sugestão é que a base "Desastres" vá além da academia, e dentro do portal possa ser combinada com os recursos de internet que já possui, e também com outros recursos, como um canal no youtube com vídeos, facilitando o acesso à informação de diferentes fontes para os públicos diversos. 


\section{Colaboradores}

V Rocha, EF Xiemenes, ML Carvalho, TMA Alpino e CM Freitas participaram igualmente de todas as etapas de elaboração do artigo.

\section{Referências}

1. Centro Latino-Americano e do Caribe de Informação em Ciências da Saúde (Bireme), Organização Pan-americana de Saúde (OPAS), Organização Mundial de Saúde (OMS). Guia da BVS 2011. São Paulo: Bireme, OPAS, OMS; 2011.

2. Silva MRB, Ferla L, Gallian DMC. Uma biblioteca sem paredes: história da criação da Bireme. Hist. cienc. saude-Manguinhos 2006; 13(1):91-112.

3. Alves FP. Informação científica, educação médica e políticas de saúde: a Organização Pan- Americana da Saúde e a criação da Biblioteca Regional de Medicina Bireme. Cien Saude Colet 2008; 13(3):899-908.

4. Biblioteca Virtual em Saúde (BVS). Comunicação pessoal recebida pelo portal da BVS, 20 de setembro de 2013. [via correio eletrônico].

5. Center for Research on the Epidemiology of Disasters (CRED) [internet]. Brussels: School of Public Health, Université Catholique de Louvain [acessado 2013 nov 16]. Disponível em: http://www.cred.be

6. Minayo MCS, Deslandes SF, Gomes R. Pesquisa social: teoria, método e criatividade. 30 a ed. Petrópolis: Vozes; 2011.

7. Organización Panamericana de la Salud (OPAS). La Salud Pública en las Américas - Nuevos Conceptos, Análisis del Desempeño, Bases para la Acción. Washington DC: OPAS; 2002.

8. Narváez L, Lavell AN, Ortega GP. La Gestión del Riesgo de Desastres: un enfoque basado en procesos. Lima: Secretaria General de la Comunidad Andina; 2009.

9. Centro de Conocimiento en Salud Pública y Desastres [internet]. Washington: Organizacíon Panamericana de la Salud. [acessado 2013 nov 13]. Disponível em: http://www.saludydesastres.info/index.php?option= com_content\&view $=$ category\&layout $=$ blog\&id $=100 \&$ Item id $=602 \&$ lang $=$ es

10. Scielo Saúde Pública [internet]. Biblioteca eletrônica online de revistas científicas em saúde pública [acessado 2014 jan 27]. Disponível em: http://www.scielosp. org

11. Instituto Nacional da Propriedade Industrial (INPI). Brasil, atuais desafios e tendências da inovação. Relatório 2013. Rio de Janeiro: INPI; 2013 [acessado 2014 fev 14]. Disponível em: http://www.inpi.gov.br/images/docs/brazil_-_current_innovation_trends_and_ challenges_final_091313_pt-br.pdf

12. Stanke C, Kerac M, Prudhomme C, Medlock J, Murray V. Health effects of drought: a systematic review of the evidence. PLoS Curr 2013; 5(5):1-38.

13. Freitas CM, Ximenes EF. Enchentes e saúde pública uma questão na literatura científica recente das causas, consequências e respostas para prevenção e mitigação. Cien Saude Colet 2012; 17(6):1601-1605.

14. Perú. Gobierno Regional Cusco. Guía de mitigación en agua y saneamiento rural-Perú. Dirección Regional de Vivienda, Construcción y Saneamiento del Cusco. Cusco: Agencia Suiza para el Desarrollo y la Cooperación (COSUDE), CARE Perú, Proyecto Saneamiento Ambiental Básico en la Sierra Sur (SANBASUR); 2011.

15. Estrategia Internacional para la Reducción de Desastres de las Naciones Unidas (EIRD/ONU). Marco de Acción de Hyogo 2005-2015. Aumento de la resiliencia de las naciones y las comunidades ante los desastres: Introducción al Marco de Acción de Hyogo. Geneva: EIRD; 2005. 
16. International Strategy for Disaster Reduction (ISDR). Reducing Disaster Risks through Science. Geneva: United Nations; 2009.

17. International Panel on Climate Change. Climate Change 2007: Impacts, Adaptation and Vulnerability Outline. Geneva: WMO,UNEP; 2007

18. Comissão Mundial Sobre Meio Ambiente e Desenvolvimento (CNUMAD). Nosso Futuro Comum. Rio de Janeiro: Editora da Fundação Getúlio Vargas; 1988.

19. Brasil. Senado Federal. Conferência das Nações Unidas sobre Meio Ambiente e Desenvolvimento: Agenda 21. Brasília: Subsecretaria de Edições técnicas; 1996.

20. United Nations Conference on Sustainable Development (UNCSD). Disaster Risk Reduction and Resilience Building [internet].2012 [acessado 2012 jan 12]:[4 p.]. Disponível em: http://www.uncsd2012. org /rio20/index.php?page $=$ view $\& n r=225 \&$ type $=400$ $\&$ menu $=45$

21. Freitas CM. A produção científica sobre o ambiente na saúde coletiva. Cad Saude Publica 2005; 21(3):679-701.

22. Camponogara S, Kirchhof ALC, Ramos FRS. Uma revisão sistemática sobre a produção científica com ênfase na relação entre saúde e meio ambiente. Cien Saude Colet 2008; 13(2):427-439.

23. Kasperson RE. Science and Disaster Reduction. Int. J. Disaster Risk Sci [periódico na internet]. 2010 [acessado 2014 fev 13]; 1(1): [3-9 p.]. Disponível em: http://link.springer.com/article/10.3974/j.issn.20950055.2010.01.002\#page-1

24. Freitas CM, Carvalho ML, Ximenes EF, Arraes EF, Gomes JO. Vulnerabilidade socioambiental, redução do risco de desastres e construção da resiliência - lições do terremoto no Haiti e das chuvas fortes na Região Serrana, Brasil. Cien Saude Colet 2012; 17(6):1577-1586.

25. United Nations Conference on Sustainable Development (UNCSD). Disaster-resilient Societies - Facts and figures [internet] 2012 [acessado 2014 jan 12]: [cerca de 2 p.]. Disponível em: http://www.un.org/en/sustainable future/disasters.shtml

26. Packer AL, Tardelli AO, Castro RCF. A distribuição do conhecimento científico público em informação, comunicação e informática em saúde indexado nas bases de dados Medline e Lilacs. Cien Saude Colet 2007; 12(3):587-599.

Artigo apresentado em 13/03/2014

Aprovado em 18/06/2014

Versão final apresentada em 20/06/2014 